\title{
Tech-X Corporation
}

Final Report: DE-FG03-01ER83313

September 16, 2005

\author{
Final Report \\ SBIR Phase II project, contract number DE-FG03-01ER 83313 \\ " ParSEC - Parallel Simulation of Electron Cooling " \\ David Bruhwiler, Principal Investigator
}

1 One Page Summary

\subsection{PURPOSE AND Motivation}

The Department of Energy has plans, during the next two or three years, to design an electron cooling section for the collider ring at RHIC (Relativistic Heavy Ion Collider) [1]. Located at Brookhaven National Laboratory (BNL), RHIC is the premier nuclear physics facility. The new cooling section would be part of a proposed luminosity upgrade [2] for RHIC. This electron cooling section will be different from previous electron cooling facilities in three fundamental ways. First, the electron energy will be $50 \mathrm{MeV}$, as opposed to 100 's of $\mathrm{keV}$ (or $4 \mathrm{MeV}$ for the electron cooling system now operating at Fermilab [3]). Second, both the electron beam and the ion beam will be bunched, rather than being essentially continuous. Third, the cooling will take place in a collider rather than in a storage ring.

Analytical work, in combination with the use and further development of the semi-analytical codes BETACOOL [4,5] and SimCool [6,7] are being pursued at BNL [8] and at other laboratories around the world. However, there is a growing consensus in the field that highfidelity 3-D particle simulations are required to fully understand the critical cooling physics issues in this new regime.

\subsection{RESEARCH AND RESULTS}

Simulations of the friction coefficient, using the VORPAL code [9], for single gold ions passing once through the interaction region, have been compared with theoretical calculations [10,11], and the results have been presented in conference proceedings papers $[8,12,13,14]$ and presentations $[15,16,17]$. Charged particles are advanced using a fourth-order Hermite predictorcorrector algorithm [18]. The fields in the beam frame are obtained from direct calculation of Coulomb's law, which is more efficient than multipole-type algorithms for less than $\sim 10^{6}$ particles. Because the interaction time is so short, it is necessary to suppress the diffusive aspect of the ion dynamics through the careful use of positrons in the simulations, and to run 100's of simulations with the same physical parameters but with different "seeds" for the particle loading.

\subsection{POTENTIAL APPLICATIONS}

VORPAL can now be used to simulate other electron cooling facilities around the world, and it is also suitable for other accelerator modeling applications of direct interest to the Department of Energy. For example: a) the Boersch effect in transport of strongly-magnetized electron beams for electron cooling sections, b) the intrabeam scattering (IBS) effect in heavy ion accelerators, c) the formation of crystaline beams and d) target physics for heavy-ion fusion (HIF). 


\section{Tech-X Corporation}

\section{Table of Contents}

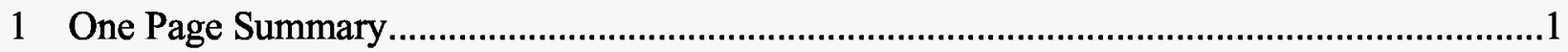

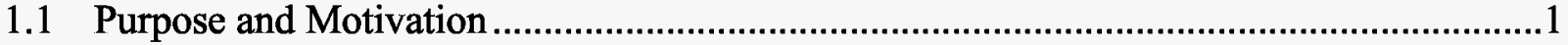

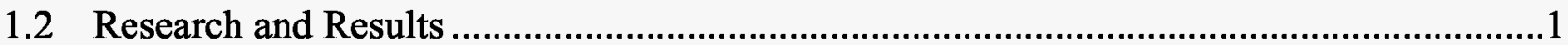

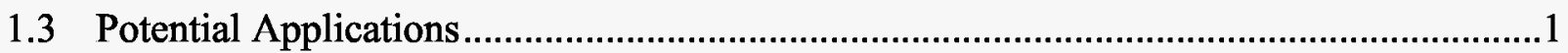

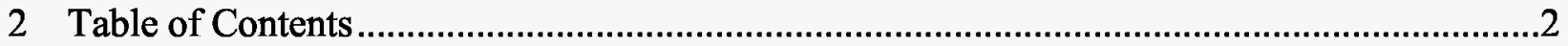

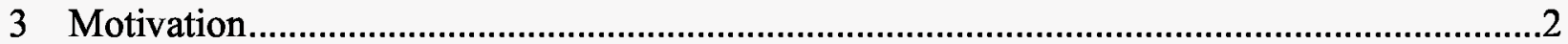

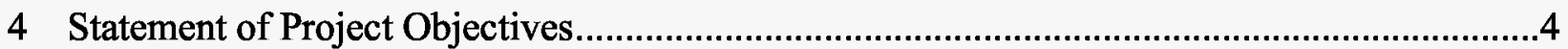

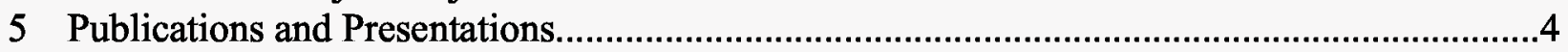

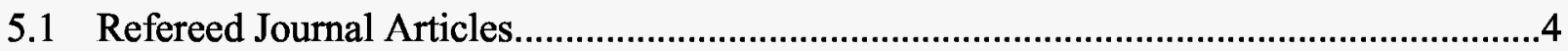

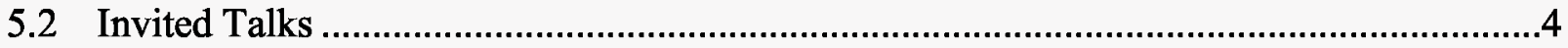

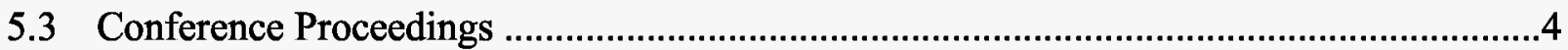

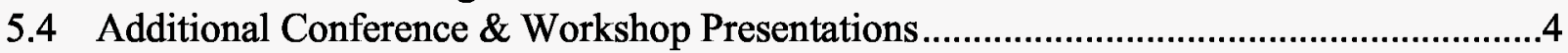

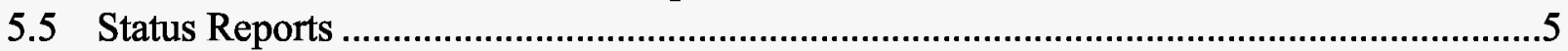

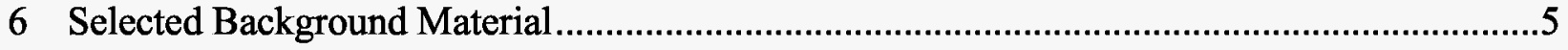

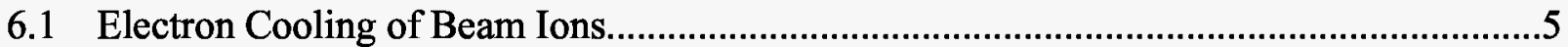

6.2 VORPAL - A Parallel, Multi-Dimension PIC and Fluid Code .......................................

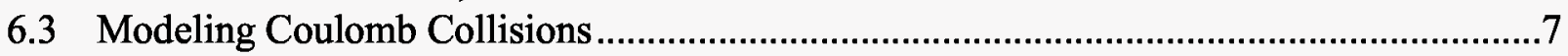

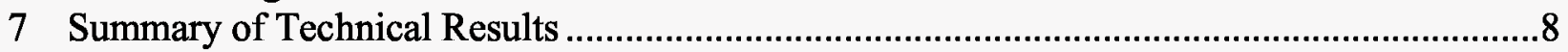

7.1 Direct Simulation of Friction Forces for Heavy Ions Interacting with a Warm Magnetized Electron Distribution .................................................................................

7.2 Numerical Studies of the Friction Force for the RHIC Electron Cooler ...........................14

7.3 The Effect of Magnetic Field Errors on Dynamical Friction in Electron Coolers .............17

8 Estimate of Technical and Economic Feasibility ...............................................................20

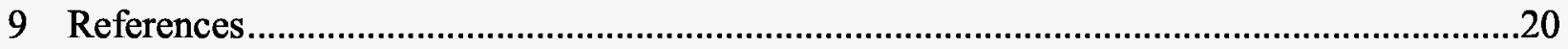

\section{Motivation}

The Relativistic Heavy Ion Collider (RHIC) [1] is arguably the most exciting nuclear physics facility in the world. Starting in June of 2000, RHIC delivered the first collisions between gold nuclei at then unprecedented energies of $100 \mathrm{GeV}$ per nucleon [19]. The primary goal of RHIC is to produce a quark-gluon plasma (QGP) through high-energy collisions of heavy ions. Analysis of the particles emerging from the QGP is expected to give scientists new insight into the conditions that occurred shortly after the big bang.

The RHIC complex of accelerators, including the $3.8 \mathrm{~km}$ circumference collider ring, is designed to accelerate a variety of ions, from protons to fully-stripped gold ions, $\mathrm{Au}^{+79}$. A luminosity upgrade is being planned for RHIC [20], in order to improve the number and quality of collisions. A key component of the upgrade is an electron cooling section [21], which will significantly improve the ion beam emittance (and, hence, the luminosity), as well as the beam lifetime. 


\section{Tech-X Corporation}

Final Report: DE-FG03-01ER83313

This electron cooling section will be different from previous electron cooling facilities in three fundamental ways [22]. First, the electron energy will be $\sim 55 \mathrm{MeV}$, as opposed to 100 's of keV (or $4 \mathrm{MeV}$ for the electron cooling system now operating at Fermilab [3,23]). Second, both the electron beam and the ion beam will be bunched, rather than being essentially continuous. Third, the cooling will take place in a collider rather than in a storage ring. These differences pose difficult challenges and much uncertainty.

Numerical studies of how emittance reduction via electron cooling will equilibrate in competition with intra-beam scattering (IBS) and other sources of emittance growth have been conducted with the semi-analytical codes BETACOOL [4,5] and SimCool [6,7]. In order to reasonably iterate for millions of turns or more, these codes use one or more reduced models to represent the electron cooling physics. BETACOOL considers a model ion distribution and each pass through the cooling section is treated as a very small emittance reduction. SimCool tracks representative particles and, with each pass through the cooling section, applies a small reduction to the relative velocities of each ion according to the expected friction force from binary Coulomb collisions with the co-propagating electrons.

Analytical expressions for the friction force on a single ion moving through a magnetized electron distribution have been published by Derbenev and Skrinsky [10] and by Parkhomchuk [11]. Parkhomchuk's formula is an empirical generalization of the theoretical friction force for unmagnetized electrons. The detailed calculation by Derbenev and Skrinsky is a generalization of the unmagnetized calculation, assuming very strong magnetization of the electrons. Both of these results implicitly assume a constant electron density, a uniform neutralizing background, and the absence of any perturbation from other ion trajectories, although Parkhomchuk's formula includes an effective velocity which may help to remove some and perhaps all of these assumptions. The results from these two very different approaches disagree with each other very strongly is some parameter regimes, while showing approximate agreement in other regimes.

This uncertainty in the magnitude of the magnetized friction force has been acceptable for existing electron cooling facilities, because they operate in a regime where the friction force is strong enough by a large margin and approximate calculations are adequate. In contrast, the proposed RHIC cooler will operate in a completely new regime, where the estimated friction force is expected to be adequate but with little margin for error. For this reason, it was necessary to develop a parallel 3D code capable of directly simulating from first principles the friction force and diffusion coefficients for parameters directly relevant to RHIC. This project used molecular dynamics techniques (i.e. simulating every single particle in the problem) to explicitly resolve close binary collisions and thus capture the friction force and the diffusion tensor with a bare minimum of physical assumptions.

The present ZDR concept for the cooling section is based on magnetized electron cooling in a high-field solenoid. This approach requires $55 \mathrm{MeV}, 20 \mathrm{nC}$ electron bunches with good emittance, strongly magnetized at the photo-cathode, with magnetization well-preserved through the linac and transport optics, leading to a $13 \mathrm{~m}$ (or longer) 2-5 Tesla superconducting solenoid. 


\section{Tech-X Corporation}

Final Report: DE-FG03-01ER83313

\section{Statement of Project Objectives}

This SBIR project was motivated by three distinct, but closely related objectives: to develop a high-performance and high-fidelity 3-D parallel simulation code for electron cooling physics, using VORPAL; to use VORPAL to model the proposed electron cooling section for the RHIC upgrade, in collaboration with BNL personnel; to develop visualization tools and documentation for VORPAL users. This report demonstrates that our objectives were accomplished.

\section{Publications and Presentations}

This project lead directly to one refereed journal articles, one invited talk, four conference proceedings papers and many presentations.

\subsection{REFEREED JOURNAL ARTICLES}

P. Messmer and D.L. Bruhwiler, "A parallel electrostatic solver for the VORPAL code," Comp. Phys. Comm. 164 (2004), p. 118.

\subsection{INVITED TALKS}

D.L. Bruhwiler, "Direct simulation of friction forces for heavy ions interacting with a warm magnetized electron distribution," Invited Talk, $33^{\text {rd }}$ ICFA Advanced Beam Dynamics Workshop on High Intensity and High Brightness Hadron Beams (Bensheim, Germany, October, 2004).

\subsection{CONFERENCE PROCEEDINGS}

D.L. Bruhwiler, R. Busby, D.T. Abell, S. Veitzer, A.V. Fedotov and V.N. Litvinenko., "The Effect of Magnetic Field Errors on Dynamical Friction in Electron Coolers," in Proc. Particle Accel. Conf. (2005), in press.

(Included below in Sec. 7.3)

A.V. Fedotov, I. Ben-Zvi, V.N. Litvinenko, D.L. Bruhwiler, D.T. Abell, R. Busby and P. Schoessow, "Numerical Studies of the Friction Force for the RHIC Electron Cooler," in Proc. Particle Accel. Conf. (2005), in press. (Included below in Sec. 7.2)

D.L. Bruhwiler, R. Busby, A.V. Fedotov, I. Ben-Zvi, J.R. Cary, P. Stoltz, A. Burov, V.N. Litvinenko, P. Messmer, D.T. Abell, C. Nieter, "Direct simulation of friction forces for heavy ions interacting with a warm magnetized electron distribution," in High Intensity and High Brightness Hadron Beams, Ed. by I. Hofmann, R.W. Hasse and J.-M. Lagniel, AIP Conf. Proc. 773 (2005), p. 394.

(Included below in Sec. 7.1)

A.V. Fedotov, I. Ben-Zvi, D.L. Bruhwiler, Yu. Eidelman, V.N. Litvinenko, N. Malitsky, I. Meshkov, A. Sidorin, A. Smirnov, G. Trubnikov, "Electron Cooling Dynamics for RHIC," in High Intensity and High Brightness Hadron Beams, Ed. by I. Hofmann, R.W. Hasse and J.M. Lagniel, AIP Conf. Proc. 773 (2005), p. 415.

\subsection{ADDITIONAL CONFERENCE \& WORKSHOP PRESENTATIONS}

R. Busby, D.L. Bruhwiler, P. Stoltz, D.T. Abell, J.R. Cary, P. Messmer, I. Ben-Zvi and A. Burov, "Direct Simulation of Friction and Diffusion Coefficients for Ions Interacting with a Magnetized Electron Distribution,” Bull. Am. Phys. Soc. 48, no. 7 (2003), p. 117. 


\section{Tech-X Corporation}

Final Report: DE-FG03-01ER83313

\subsection{STATUS REPORTS}

D.L. Bruhwiler, R. Busby, D.T. Abell, P. Messmer, P. Stoltz, J.R. Cary, A. Fedotov, I. BenZvi, V. Litvinenko and A. Burov, "Simulating Electron Cooling Physics with VORPAL Status, Current Plans \& Future Developments," presented at BNL (Dec., 2003); see URL http:/www.agsthichome.bnl.gov/eCool/workshop 1203/2 Bruhwiler VORPAL status.pdf

D.L. Bruhwiler, R. Busby, D.T. Abell, P. Messmer, P. Stoltz, J.R. Cary, A. Fedotov, I. BenZvi, V. Litvinenko and A. Burov, "Simulating Electron Cooling Physics with VORPAL Recent Results," presented at BNL (Dec., 2003); see URL htp://www.agsthichome.bnl.gov/eCool/workshop 1203/6 Bruhwiler VORPAL results.pdf

\section{Selected Background Material}

\subsection{ELECTRON COOLING OF BEAM IONS}

The concept of electron cooling for ion beams was first proposed by Budker in 1966 [24]. The general idea is to have a cold electron beam copropagate with the ion beam for some distance in one portion of a storage ring, such that some thermal energy is transferred from the ion beam to the electron beam during each pass through the cooling section. There are now on the order of 50 electron cooling sections in operation at ion storage rings around the world. Three examples include the Indiana University Cyclotron Facility (IUCF) in Bloomington, Indiana [25], the SIS heavy ion synchrotron at the Gesellschaft für Schwerionenforschung (GSI) in Darmstadt, Germany [26], and the accumulator cooler ring (ACR) at the MUSES project in Japan [27].

The electron beam must travel with the same average velocity as the ion beam. Most ion rings operate at modest beam energy, so the electron beams have only had energies on the order of a few hundred $\mathrm{KeV}$ or less. A recent development is the $4 \mathrm{MeV}$ electron beamline for cooling that is now operating at Fermi National Accelerator Laboratory (Fermilab) [3,23]. The top energy for gold ions in RHIC is on the order of $100 \mathrm{GeV}$ per nucleon, corresponding to a relativistic factor $\gamma \sim 107$. Thus, the electrons must also have $\gamma \sim 107$, corresponding to an energy of roughly $55 \mathrm{MeV}$, which necessitates the use of an electron linac.

To prevent recombination of the electrons with the $\mathrm{Au}^{+79}$ ions, the transverse electron temperature must be large - at least $400 \mathrm{eV}$. The interaction region of the RHIC facility will include a $30 \mathrm{~m}$ long solenoid, with a nominal field strength of $1 \mathrm{~T}$ or perhaps somewhat larger. In order to keep the electrons magnetized (i.e. gyroradius significantly smaller than both the beam radius and the maximum impact parameter for binary Coulomb collisions), and given the technological and funding limitations on feasible field strengths for a $30 \mathrm{~m}$ solenoid, the maximum transverse temperature that can be tolerated is of order $1,500 \mathrm{eV}$.

The fundamental property of magnetized electron cooling is that the transverse electron temperature is irrelevant to the friction force, for impact parameters ranging between the gyroradius (or Larmor radius) $r_{L}$ and the maximum value $\rho_{\max }[10]$. Sometimes $\rho_{\max }$ is the beam radius, but for RHIC it is a generalization of the Debye screening length - the electron thermal velocity is replaced by the maximum of itself and the ion velocity; also, the inverse of the electron plasma frequency is replaced by the interaction time, if this is smaller. For this range of impact parameters, the ion effectively collides with a "Larmor circle" rather than a fast electron, 


\section{Tech-X Corporation}

Final Report: DE-FG03-01ER83313

and so the strength of the cooling is governed by the longitudinal electron temperature, which is orders of magnitude smaller.

Of course, the physical picture described in the preceding paragraph is an approximation. Derbenev and Skrinsky [10] argue that there are two contributions to the friction force - the unmagnetized part, with impact parameters ranging from $\rho_{\min }$ (related to the thermal distance of closest approach or, equivalently, what is needed for right-angle scattering) to $r_{L}$; and the magnetized part, with impact parameters ranging from $r_{L}$ to $\rho_{\max }$. They then calculate the magnetized contribution in the limit of a very strong solenoidal field. For electrons with a very high transverse temperature, as is the case for RHIC, the unmagnetized contribution can be neglected. This approach requires that $\rho_{\min } \ll \mathrm{r}_{\mathrm{L}} \ll \rho_{\max }$. For RHIC parameters, the "adiabatic" Coulomb logarithm in the magnetized friction force, $\ln \left(\rho_{\max } / \mathrm{r}_{\mathrm{L}}\right)$ will be very close to unity for realistic solenoidal field values. Thus, it is not clear how well the analysis of Derbenev and Skrinsky will work for the RHIC cooler.

Parkhomchuk takes a fundamentally different approach [11], by starting with the theoretical friction force for unmagnetized cooling, and empirically generalizing the Coulomb logarithm from $\ln \left(\rho_{\max } / \rho_{\min }\right)$ to the new form $\ln \left[\left(\rho_{\max }+r_{L}+\rho_{\min }\right) /\left(r_{L}+\rho_{\min }\right)\right]$. This functional form varies smoothly over a wide range of parameters (i.e. the inequalities of the previous paragraph are not required), encompassing both weakly- and strongly-magnetized electrons, but it does not apply to the unmagnetized case, where $r_{L} \rightarrow \infty$. Parkhomchuk also includes in his formula an effective velocity, which contains a contribution from the longitudinal thermal electron velocity, from space charge effects and from magnetic field errors. It is claimed [11] that this empirical formula agrees well with experimental data from many existing cooler facilities, although it does not agree with some of the idealized numerical simulations presented in the same paper.

The analysis of Derbenev and Skrinsky neglects the effects of strong variations in the electron density, space charge fields due to differences in the electron and ion beam distributions, errors in the solenoidal magnetic field, and perturbations due to nearby and crossing trajectories of other ions. All of these effects will be present in the RHIC cooler.

\subsection{VORPAL - A PARALlel, Multi-Dimension PIC AND Fluid CODE}

The VORPAL code $[9,28]$ is under development jointly at the University of Colorado and Tech$\mathrm{X}$ Corporation for the purpose of simulating intense laser-plasma physics. VORPAL currently can model the interaction of electromagnetic fields with conducting boundaries, charged particles (of both constant and variable weight), and charged fluids. Included in the fluid implementations are both a cold (pressureless) fluid and a scalar-pressure fluid. The VORPAL framework also has field ionization production of particles, and it has a Direct Simulation Monte Carlo treatment of neutral and charged fluids, i.e., self collisions for the particle representations.

The VORPAL framework uses $\mathrm{C}++$ templating over both dimension and floating point type, so that one can build 1-D, 2-D and 3-D codes, in both single and double precision, using a single source code base. VORPAL makes use of the templated, reference-counted container classes from the OptSolve++ library [29], which was developed at Tech-X. Output is in the form of HDF5, a binary, cross-platform, self-describing data format. In addition, VORPAL was designed to have top-level interaction only through a single object. This will allow VORPAL to be incorporated into a desktop application with a GUI on Windows or Unix.

The VORPAL framework is designed for use on multiple platforms. Use of the GNU configure 


\section{Tech-X Corporation}

Final Report: DE-FG03-01ER83313

tools (automake, autoconf, configure, make) has enabled a VORPAL distribution that can be built and run on Linux and multiple UNIX platforms. In addition, VORPAL has been ported to Windows and Macintosh OS X. VORPAL can run on a parallel Beowulf cluster or on the parallel IBM SP2 through use of the Message Passing Interface (MPI). (On OS X, VORPAL works with the LAM MPI implementation.)

At present VORPAL runs in batch mode, generating output, as noted above, in the form of HDF5 files. The files can then be read by any number of visualization tools for further analysis. The VORPAL distribution comes with a number of visualization programs for carrying out this task.

VORPAL has been shown to scale very well in parallel computations. The figure below shows the parallel speedup of VORPAL as a function of the number of processors on the Seaborg machine at the National Energy Research Supercomputer Center (NERSC). The solid line, linear scaling, is very closely followed out to 4000 processors, the maximum number available at NERC. This indicates that VORPAL may be able to make effective use of the even larger number of processors soon to become available at other computing centers.

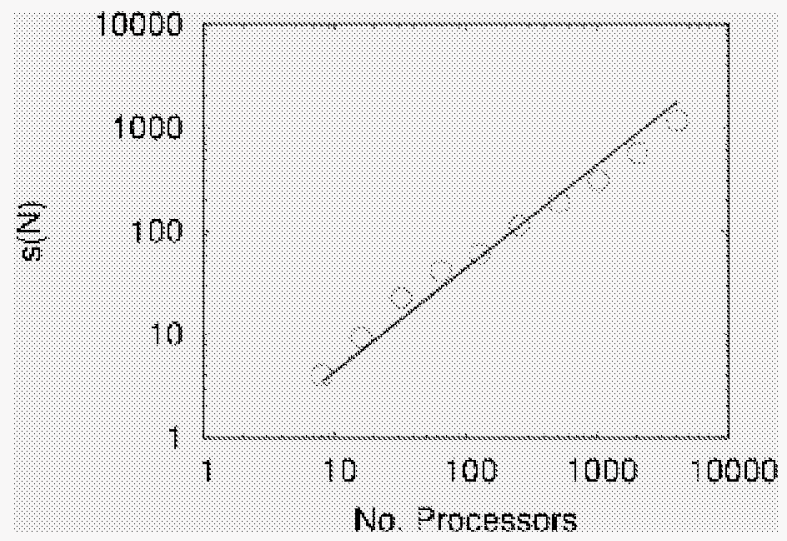

Figure 1.1.2 - 1. Speedup of VORPAL on SP3 at the National Energy Research Supercomputer Center.

The VORPAL developers (which now number more than 10) have put together an extensive website $[30,31]$ on the use of VORPAL. This web includes documentation on the building and use of VORPAL. The latter includes some preliminary documentation of input files along with documentation on data visualization and run management. The VORPAL modeling application is freely available to the research community under the CU research license, as noted at the website.

Recently VORPAL has experienced success in the form of making the cover of Nature [32]. The associated article [33] discusses the production of high-energy particle beams with narrow energy spread through the breakup of laser pulses propagating through a plasma when the are just a bit longer than the plasma wavelength. VORPAL simulations were used to help understand the physics of the experiment.

\subsection{Modeling Coulomb Collisions}

One can also dispense with the intervening grid and calculate the inter-particle coulomb forces directly. This is a classical N-body problem, in which one simulates the evolution of a system of $\mathrm{N}$ particles, and the force exerted on each particle arises due to its interaction with all the other 


\section{Tech-X Corporation}

Final Report: DE-FG03-01ER83313

particles in the system. N-body algorithms have important applications in other fields of computational science, including biophysics, astrophysics and molecular dynamics.

When all pair wise forces are computed directly, $\mathrm{O}\left(\mathrm{N}^{2}\right)$ operations are required for each time step, making this approach numerically intensive in the extreme. For biophysical applications like protein folding, where the charged particles remain well-separated and one can use a fixed time step, there are three types of tree-based algorithms [34] that dramatically improve this scaling: the Barnes-Hut algorithm [35], the fast multipole algorithm (FMA) [36,37], and the multipole tree algorithm [38]. For astrophysical applications, where stars are treated as gravitating point masses and very close binary collisions must be resolved, there is a need for aggressive variation of the time step for each "particle", and it has been found that the Hermite algorithm mentioned in the previous section [18] is more efficient than a fixed-time-step multipole algorithm for greater than $10^{6}$ particles, in spite of the unfavorable scaling with particle number.

Primitive N-body simulations were tested in early accelerator codes and were found to be unacceptably noisy, due to the problem of artificially close collisions. In an artificially close collision, two charged particles approach each other very closely due to the finite time step and, consequently, undergo an unrealistically strong collision. The PIC algorithm avoids this problem at the expense of losing Coulomb collisions altogether. It is these close particle-toparticle interactions which are critical to correctly modeling the physics of electron cooling. This problem has now been solved by implementing within VORPAL the variable-time-step Hermite algorithm originally developed by the astrophysical dynamics community [18] (with some generalization for including a B-field) [14].

\section{$7 \quad$ Summary of Technical Results}

Our technical results are succinctly summarized in three proceedings papers. Rather than duplicate that material here, we have inserted these papers directly:

7.1 "Direct Simulation of Friction Forces for Heavy Ions..." begins on p. 9.

7.2 "Numerical studies of the friction force for the RHIC Electron Cooler" begins on p. 14.

7.3 "The Effect of Magnetic Field Errors on Dynamical Friction..." begins on p. 17.

The full references to these papers can be found above in Sec. 5.3. 


\title{
Direct Simulation of Friction Forces for Heavy Ions Interacting with a Warm Magnetized Electron Distribution
}

\author{
D.L. Bruhwiler ${ }^{1}$, R. Busby ${ }^{1}$, A.V. Fedotov ${ }^{2}$, I. Ben-Zvi ${ }^{2}$, J.R. Cary ${ }^{1,3}$, P. Stoltz ${ }^{1}$, \\ A. Burov ${ }^{4}$, V.N. Litvinenko ${ }^{2}$, P. Messmer ${ }^{1}$, D. Abell ${ }^{1}$ and C. Nieter ${ }^{1}$ \\ ${ }^{1}$ Tech-X Corp., Boulder, CO 80303, USA \\ ${ }^{2}$ Brookhaven National Laboratory, Upton, NY 11973, USA \\ ${ }^{3}$ University of Colorado, Boulder, CO 80309, USA \\ ${ }^{4}$ Fermilab, Batavia, IL 60510, USA
}

\begin{abstract}
A proposed luminosity upgrade to RHIC includes a novel electron cooling section [1], which would use $55 \mathrm{MeV}$ electrons to cool fully-ionized $100 \mathrm{GeV} /$ nucleon gold ions. High-current bunched electron beams are required for the RHIC cooler, resulting in very high transverse temperatures and relatively low values for the magnetized cooling logarithm. The accuracy of analytical formulae in this regime requires careful examination. Simulations of the friction coefficient, using the VORPAL code [2], for single gold ions passing once through the interaction region, are compared with theoretical calculations $[3,4]$. Charged particles are advanced using a fourth-order Hermite predictor-corrector algorithm [5]. The fields in the beam frame are obtained from direct calculation of Coulomb's law, which is more efficient than multipole-type algorithms for less than $\sim 10^{6}$ particles. Because the interaction time is so short, it is necessary to suppress the diffusive aspect of the ion dynamics through the careful use of positrons in the simulations.
\end{abstract}

\section{INTRODUCTION}

The Relativistic Heavy Ion Collider (RHIC) [6] delivered the first collisions between gold nuclei in 2000 at then unprecedented energies of $100 \mathrm{GeV}$ per nucleon [7]. RHIC is designed to accelerate a wide variety of ions, from protons to fully-stripped gold, $\mathrm{Au}^{+79}$. A luminosity upgrade is being planned for RHIC [1], in order to improve the number and quality of collisions. A key component of the upgrade is an electron cooling section [8], which will significantly improve the ion beam luminosity and lifetime.

The RHIC electron cooler will be fundamentally different from existing facilities $[1,9]$. The electron energy must be $\sim 55 \mathrm{MeV}$, requiring $\mathrm{rf}$ acceleration of a high-charge $(\sim 20 \mathrm{nC})$ magnetized electron bunch, with orders-of-magnitude larger temperatures then has been previously considered. Even with a solenoidal magnetic field as large as $5 \mathrm{~T}$, the relevant Coulomb logarithm will be of order unity, so analytical models are only marginally applicable.
Analytical expressions for the friction force on a single ion moving through a magnetized electron distribution have been published by Derbenev and Skrinsky [3,10], Meshkov [11], Parkhomchuk [4] and Toepffer [12], among others. Parkhomchuk's formula is an empirical generalization of the theoretical friction force for unmagnetized electrons. The semi-numerical results of Toepffer, which have not yet been compared with others, will not be discussed further here.

These calculations implicitly assume a constant electron density, a uniform neutralizing background, the absence of any perturbation from other ion trajectories, and a constant longitudinal magnetic field. Parkhomchuk's formula includes an effective velocity which may help to remove some of these assumptions in a parametric fashion. The Derbenev-SkrinskyMeshkov (DSM) and Parkhomchuk formulas disagree strongly in some parameter regimes, while showing approximate (roughly factor of two) agreement in other regimes. These formulas are applied in the beam frame, assuming nonrelativistic particle velocities and electrostatic particle-field dynamics. 
Order of magnitude calculations of the magnetized friction force has been acceptable for existing electron cooling facilities, because they operate in a regime where the friction force is strong enough by a large margin. In contrast, the proposed RHIC cooler will operate in a completely new regime, where the estimated friction force is expected to be adequate but with little margin for error.

Hence, we are developing a parallel 3D code capable of directly simulating from first principles the magnetized friction force and diffusion coefficients for parameters relevant to RHIC. This project is using molecular dynamics techniques (i.e. simulating every particle in the problem) to explicitly resolve close binary collisions and thus capture the friction force and the diffusion tensor with a bare minimum of physical assumptions $[9,13]$.

These on-going simulations show great promise for resolving ambiguities in the theoretical understanding of the magnetized friction force under idealized conditions, and also for determining quantitatively the effect of complicating factors, such as the impact of errors in the solenoidal magnetic field.

\section{NUMERICAL APPROACH}

When all pairwise forces are computed directly for $\mathrm{N}$ particles, $\mathbf{O}\left(\mathrm{N}^{2}\right)$ operations are required for each time step, making this approach numerically intensive. For biophysical applications like protein folding, where the charged particles remain well-separated and one can use a fixed time step, there are three types of tree-based algorithms [14] that dramatically improve this scaling: the Barnes-Hut algorithm [15], the fast multipole algorithm (FMA) [16,17], and the multipole tree algorithm [18].

For astrophysical applications, where stars are treated as gravitating point masses and very close binary collisions must be resolved, there is a need for aggressive variation of the time step for each "particle". It has been found that a specialized $4^{\text {th }}$ order predictor-corrector algorithm [19,5], used with the $\mathbf{O}\left(\mathrm{N}^{2}\right)$ field calculation, is more efficient than a fixed-time-step multipole algorithm for less than $10^{6}$ particles, in spite of the unfavorable scaling.

It is these close particle-to-particle interactions which are critical to correctly modeling the physics of electron cooling, especially in regimes where the relevant Coulomb logarithm is of order unity. Thus, we have generalized the $4^{\text {th }}$-order algorithm of Makino \& Aarseth to accommodate charged particles in a magnetic field [13] and implemented it within the parallel simulation code VORPAL [2]. New positions and velocities for the $i^{\text {th }}$ particle are predicted from a Taylor expansion in time, using the following acceleration and its time derivative:

$$
\begin{aligned}
\frac{m_{i} \mathbf{a}_{i}}{q_{i}} & =\mathbf{v}_{i} \times \mathbf{B}+\frac{1}{4 \pi \varepsilon_{0}} \sum_{j} \frac{q_{j} \mathbf{r}_{i j}}{\left(r_{i j}^{2}+r_{c}^{2}\right)^{3 / 2}} \\
\frac{m_{i} \dot{\mathbf{a}}_{i}}{q_{i}} & =\mathbf{a}_{i} \times \mathbf{B}+\sum_{j} \frac{q_{j}}{4 \pi \varepsilon_{0}}\left[\frac{\mathbf{v}_{i j}}{\left(r_{i j}^{2}+r_{c}^{2}\right)^{3 / 2}}+\frac{3\left(\mathbf{v}_{i j} \cdot \mathbf{r}_{i j}\right) \mathbf{r}_{i j}}{\left(r_{i j}^{2}+r_{c}^{2}\right)^{5 / 2}}\right]
\end{aligned}
$$

where $\mathbf{r}_{\mathrm{ij}}$ and $\mathbf{v}_{\mathrm{ij}}$ are the relative positions and velocities of particles $i$ and $j$, and the "cloud radius" $r_{\mathrm{c}}$ is typically zero.

The more complicated "corrector" step follows Ref. [5], except that retaining $4^{\text {th }}$-order accuracy requires the $v x \mathbf{B}$ force to be recalculated with the predicted velocity, and one of the coefficients must be changed for terms that include B. Particles are binned in time step levels that differ by factors of two, and they are advanced in an order that minimizes the time separation between different populations. After each particle is pushed, a new time step is calculated and, if necessary, particles are moved to a new level. Whenever a corrector step is applied to one population of particles, all other populations are brought temporarily to the same time with a predictor step (i.e. no force calculation) and then the force is calculated only for those particles in the active population. These details are beyond the scope of the present paper and will be clarified in future work.

\section{FRICTION \& DIFFUSION FOR B=0}

Our implementation of this algorithm was first applied to an ion moving through an unmagnetized plasma. The dynamical friction force due to the plasma electrons is given $[20,21,22]$ by Eq. (2a):

$$
\begin{gathered}
\mathbf{F}=-\omega_{p e}^{2} \frac{(Z e)^{2}}{4 \pi \varepsilon_{0}} \ln \left(\frac{\rho_{\max }}{\rho_{\min }}\right) \frac{\mathbf{V}_{i o n}}{V_{i o n}^{3}} \Psi(\alpha) \\
\rho_{\min }=\left(Z e^{2} / 4 \pi \varepsilon_{0}\right) / m_{e} V_{r e l}^{2} \quad \rho_{\max }=V_{r e l} / \max \left(\omega_{p e}, 1 / \tau\right) \\
V_{r e l}=\max \left(V_{i o n}, V_{e, r m s}\right) \quad \omega_{p e}=\sqrt{n_{e} e^{2} / \varepsilon_{0} m_{e}} \\
\Psi(\alpha)=\frac{2}{\sqrt{\pi}} \int_{0}^{\alpha} t^{1 / 2} e^{-t} d t \quad \alpha=V_{i o n}^{2} / 2 V_{e, r m s}^{2}
\end{gathered}
$$

Budker [23] first suggested that this friction could be used to cool heavy ions in a storage ring by having them co-propagate repeatedly with cold electrons.

For RHIC, the interaction time is $\tau=L / \gamma c \sim 0.4 n s$, where $L \sim 13 \mathrm{~m}$ is the length of a solenoid magnet (for a 
design with two solenoids of $13 \mathrm{~m}$ each [8]), $\gamma \sim 107$ is the relativistic factor, and $\mathrm{c}$ is the speed of light. For a beam frame electron density of $n_{\mathrm{e}} \sim 1 \times 10^{15} \mathrm{~cm}^{-3}$, the plasma frequency is $\omega_{\mathrm{pe}} \sim 1 \times 10^{9} \mathrm{rad} / \mathrm{m}$. In this regime, the interaction time is smaller than a plasma period, so the electrons don't have time to screen the ion. Thus, we choose to neglect the e-/e- interactions, resulting in shorter simulation times that scale as $\mathbf{O}\left(\mathrm{N}_{\text {ion }}{ }^{*} \mathrm{~N}_{\mathrm{e}}\right)$.

For these very short interaction times, the rms spread in velocity due to diffusion is of the same order of magnitude as the velocity drag due to friction:

$$
d V_{f}=-|\mathbf{F}| \tau / m_{i o n} \quad \delta V_{\|, r m s}=\sqrt{\tau D_{\|}}
$$

where the parallel $D_{\|}$and perpendicular diffusion coefficients have different functional forms $[20,21$, 22]. This is shown in Fig. 1, for an electron thermal velocity of $V_{\mathrm{e}, \mathrm{rms}}=4 \times 10^{4} \mathrm{~m} / \mathrm{s}$, and a variety of initial ion velocities. This makes it difficult to accurately extract the friction force from simulated ion velocities. In the limit of millions of interactions, however, as will occur in RHIC, the diffusive dynamics becomes negligible, because it scales as the square root of the number of interactions, while friction scales linearly.

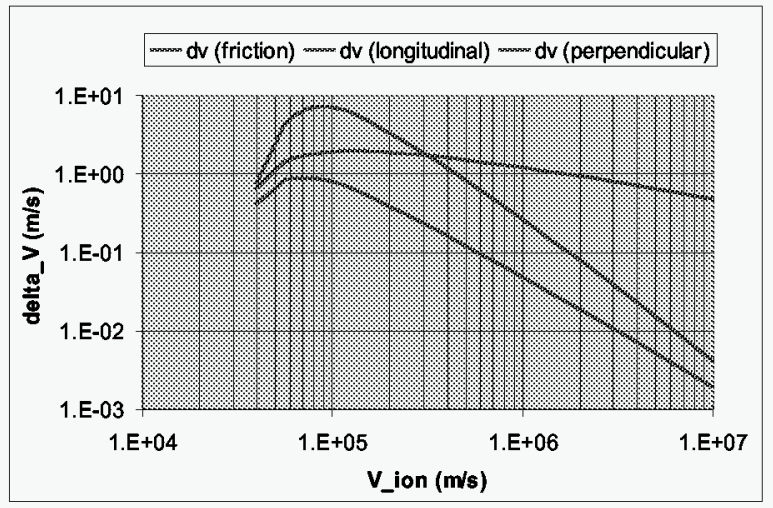

FIGURE 1. The rms spread of longitudinal (red) and perpendicular (green) velocity kicks are comparable to the dynamic velocity drag (blue) for brief interactions.

In the limit of an ideal plasma, $Z<<N_{\mathrm{D}}$, where $Z$ is the ion charge number and $N_{\mathrm{D}}$ is the number of electrons in a Debye sphere, the dynamical friction due to positrons is identical to that of electrons. If half of the electrons are replaced by positrons, the friction force is unchanged. Given a distribution of initially correlated e-/e+ pairs (same position and velocity), the diffusive kicks from each e- will be approximately cancelled by the corresponding $\mathrm{e}+$.

Figure 2 shows the dynamics of a single $\mathrm{Au}^{+79}$ ion, with correlated e-/e+ pairs used to suppress diffusion. The velocity drag due to friction is seen in the bottom frame. Here, we have $\tau=0.935 \mathrm{~ns}(L=30 \mathrm{~m}) ; \mathrm{B}=1 \mathrm{~T}$; $n_{\mathrm{e}}=2 \times 10^{15} \mathrm{~m}^{-3} ; \mathrm{V}_{\mathrm{e}, \mathrm{rms}}=4 \times 10^{4} \mathrm{~m} / \mathrm{s}$ (same parameters as in Fig. 1), for initial ion velocity $V_{\text {ion }}=6 \times 10^{5} \mathrm{~m} / \mathrm{s}$.
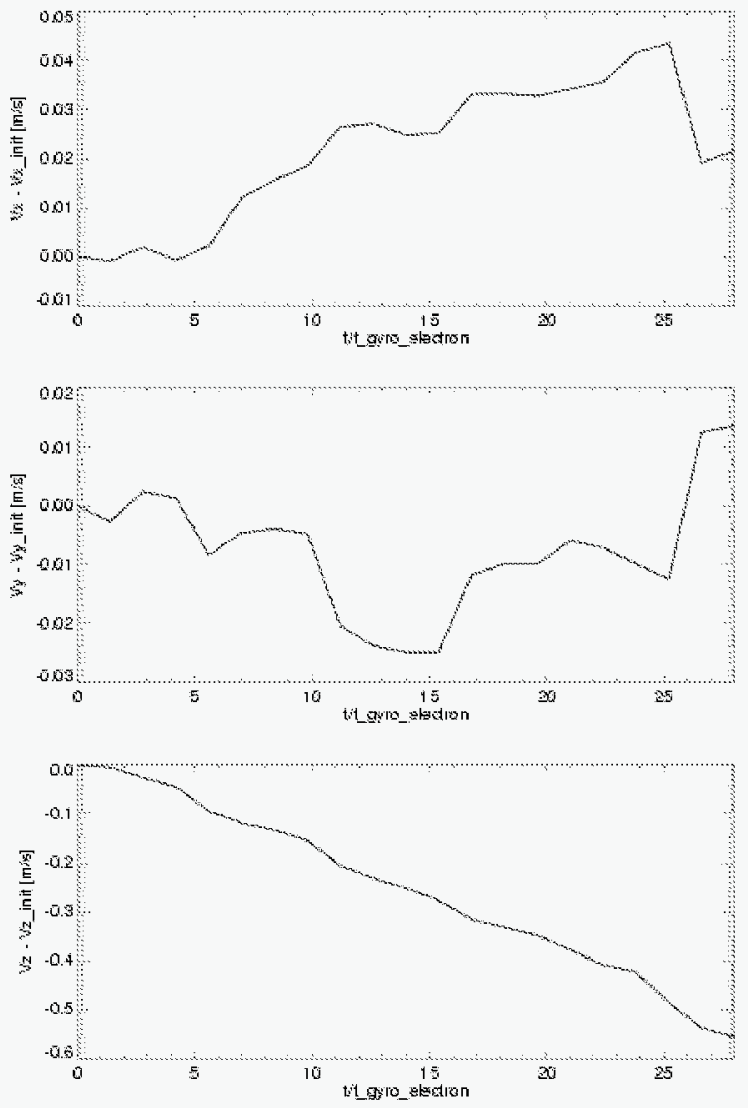

FIGURE 2. The change in transverse $\left(V_{\mathrm{x}}, V_{\mathrm{y}}\right)$ and longitudinal $\left(V_{\mathrm{z}}\right)$ velocities for an $\mathrm{Au}^{+79}$ ion, with initial velocity $V_{\mathrm{z}}=6.5 \times 10^{4}$. The diffusive dynamics is artificially suppressed by using correlated e-/e+ pairs.

Table 1 shows a comparison of the frictional velocity drag as simulated by VORPAL and predicted by theory. The agreement is good when the Coulomb log is large, and poor when it is of order unity or smaller.

TABLE 1. Unmagnetized velocity drag due to friction

\begin{tabular}{cccc}
\hline$V_{\text {ion }}(\mathrm{m} / \mathrm{s})$ & $\ln \left(\rho_{\text {max }} / \rho_{\text {min }}\right)$ & $d V_{f}($ theory $)$ & $d V_{f}($ VORPAL $)$ \\
\hline $6.0 \times 10^{5}$ & 8 & -0.61 & -0.62 \\
$2.0 \times 10^{5}$ & 5 & -3.3 & -2.5 \\
$0.4 \times 10^{5}$ & 0.2 & -0.8 & -0.02
\end{tabular}




\section{MAGNETIZED FRICTION THEORY}

The transverse rms electron velocity $V_{\mathrm{e}, \mathrm{rms}, \perp}$ in the proposed RHIC cooler will be much larger than the ion velocity, so dynamical friction will be negligible, unless the electrons are strongly magnetized. For ion velocities ranging from the longitudinal $\mathrm{rms}$ electron

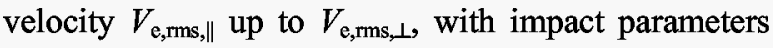
larger than the electron Larmor radius $r_{\mathrm{L}}$, the ion effectively interacts with a ring of charge, rather than a fast electron. This results in a much stronger exchange of energy.

The formulas presented by Derbenev, Skrinsky and Meshkov (DSM) are relevant to this parameter regime:

$$
\begin{aligned}
& \mathrm{F}_{\|}^{D S M}=-\frac{3}{2} \omega_{p e}^{2} \frac{(Z e)^{2}}{4 \pi \varepsilon_{0}}\left[\ln \left(\frac{\rho_{\max }^{D S M}}{\rho_{\min }^{D S M}}\right)\left(\frac{V_{\perp}}{V_{i o n}}\right)^{2}+\frac{2}{3}\right] \frac{V_{\|}}{V_{i o n}^{3}} \\
& \mathrm{~F}_{\perp}^{D S M}=-\omega_{p e}^{2} \frac{(Z e)^{2}}{4 \pi \varepsilon_{0}} \ln \left(\frac{\rho_{\max }^{D S M}}{\rho_{\min }^{D S M}}\right) \frac{\left(0.5 V_{\perp}^{2}-V_{\|}^{2}\right)}{V_{i o n}^{2}} \frac{V_{\perp}}{V_{i o n}^{3}} \\
& \rho_{\min }^{D S M}=\max \left(r_{L}, \rho_{\min }\right) \quad r_{L}=V_{r m s, e, \perp} / \Omega_{L} \\
& \rho_{\max }^{D S M}=V_{r e l}^{D S M} / \max \left(\omega_{p e}, 1 / \tau\right) \\
& V_{r e l}^{D S M}=\max \left(V_{i o n}, V_{e, r m s, \|}\right) \quad V_{i o n}^{2}=V_{\|}^{2}+V_{\perp}^{2}
\end{aligned}
$$

These results are valid in the limit that the magnetized Coulomb log is large. Hence, they are of limited value in the limit of poor magnetization, when the maximum impact parameter is not much larger than the Larmor radius.

In contrast, Parkhomchuk presents a parametric formula - a generalization of the unmagnetized result, which is predicted [4] to agree reasonably well with experiments for a wide range of parameters:

$$
\begin{gathered}
\mathbf{F}^{P}=-\frac{1}{\pi} \omega_{p e}^{2} \frac{(Z e)^{2}}{4 \pi \varepsilon_{0}} \ln \left(\frac{\rho_{\max }^{P}+\rho_{\min }^{P}+r_{L}}{\rho_{\min }^{P}+r_{L}}\right) \frac{\mathbf{V}_{i o n}}{\left(V_{i o n}^{2}+V_{e f f}^{2}\right)^{3 / 2}} \\
\rho_{\min }^{P}=\left(Z e^{2} / 4 \pi \varepsilon_{0}\right) / m_{e} V_{i o n}^{2} \\
\rho_{\max }^{P}=V_{i o n} / \max \left(\omega_{p e}, 1 / \tau\right) \\
V_{e f f}^{2}=V_{e, r m s, \|}^{2}+\Delta V_{\perp e}^{2}
\end{gathered}
$$

The subtle differences in the definitions of the min and max impact parameters, as compared to DSM or the unmagnetized case, lead to very different behavior. In the limit of weak magnetization, or small ion velocity, the ordering in size of the three impact parameters can change, while the log remains well defined. The new parameter $\Delta V_{\perp e}$, an effective transverse velocity of the charge rings, is used to capture the effects of transverse $\mathbf{E}$ and $\mathbf{B}$ fields.

Figure 3 compares the predictions of Parkhomchuk and DSM for purely longitudinal and transverse ion motion. One can see the unmagnetized contribution to friction is negligible. Here, we have $\tau=0.935 \mathrm{~ns}$ ( $L=30$ $\mathrm{m}) ; \mathrm{B}=5 \mathrm{~T} ; n_{\mathrm{e}}=2 \times 10^{15} \mathrm{~m}^{-3} ; V_{\mathrm{e}, \mathrm{rms}, \perp}=8 \times 10^{6} \mathrm{~m} / \mathrm{s} ;$ and $V_{\mathrm{e}, \mathrm{rms}, \|}=1 \times 10^{5} \mathrm{~m} / \mathrm{s}$. The VORPAL simulations (red squares) show reasonable agreement with the DSM and Parkhomchuk formulas.

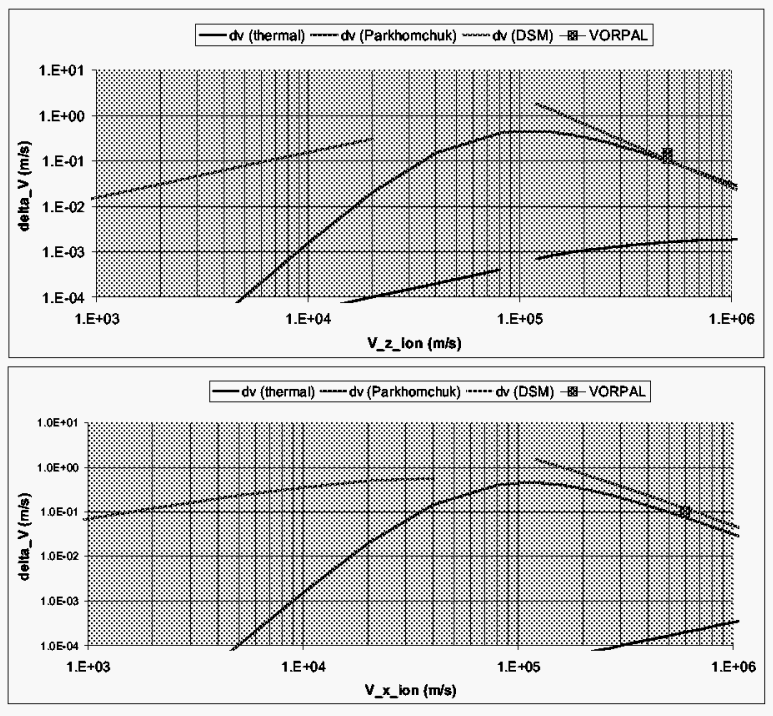

FIGURE 3. The velocity drag due to dynamical friction, according to DSM (green), Parkhomchuk (blue), unmagnetized theory (black) and VORPAL simulations (red), for the case of ion motion along the magnetic field (top) and perpendicular (bottom).

The parameter $\Delta V_{\perp \mathrm{e}}$, which is zero in Fig. 3, can be chosen to model transverse velocity kicks of the charged electron rings driven by misalignments in the solenoidal fields. The Parkhomchuk model indicates that any field misalignments will reduce the friction force. Recent work [24] indicates that the effects are not so straightforward. The DSM model cannot include any effects due to misalignments.

Figure 4 shows the ion dynamics from a VORPAL simulation for these parameters, with perpendicular initial ion velocity $V_{\text {ion }}=V_{x}=6 \times 10^{5} \mathrm{~m} / \mathrm{s}$. Other parameters are the same as in Fig. 3. Correlated e-/e+ pairs were used to suppress diffusion. The top frame shows the resulting velocity drag of the $\mathrm{Au}^{+79}$ ion. 

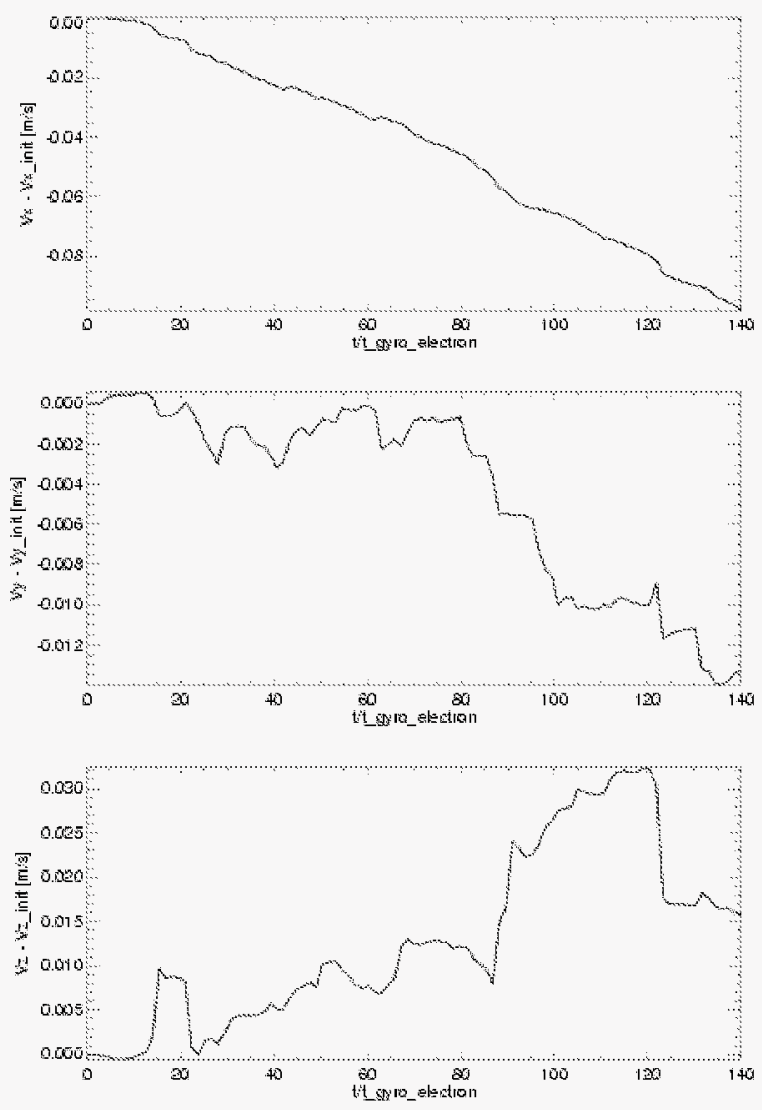

FIGURE 4. The change in transverse $\left(V_{\mathrm{x}}, V_{\mathrm{y}}\right)$ and longitudinal $\left(V_{z}\right)$ velocities for an $\mathrm{Au}^{+79}$ ion, with initial velocity $V_{\mathrm{x}}=6 \times 10^{5}$. The diffusive dynamics is artificially suppressed by using correlated e-/e+ pairs.

\section{SUMMARY}

A unique molecular dynamics algorithm has been implemented to study the dynamical friction and diffusion of ions moving through electrons and positrons. Binary coulomb collisions are explicitly resolved, in order to minimize the number of assumptions and approximations being made. Careful testing indicates that the algorithm is working well for magnetized and unmagnetized cases that are relevant to the proposed electron cooling section for RHIC.

Ongoing efforts are directed towards parameter studies to distinguish competing analytical formulas. Future work will address the critical issue of magnetic field errors and the extent to which these reduce the friction force and, hence, the effectiveness of the cooling. Efforts to reduce run time and improve parallel performance are also continuing.

\section{ACKNOWLEDGMENTS}

We thank S. Aarseth, O. Boine-Frankenheim, Ya. Derbenev, P. Hut, J. Makino, S. McMillan, R. Spurzem, V. Ziemann, and the Accelerator Physics Group of the RHIC Electron Cooling Project for helpful discussions. This work was supported by the U.S. Dept. of Energy under grants DE-FG0301ER83313, DE-FC02-01ER41178 and DE-FG0395ER40926 and by Tech-X Corp. This research used resources of the National Energy Research Scientific Computing Center, which is supported by the U.S. Dept. of Energy under contract DE-AC03-76SF00098.

\section{REFERENCES}

1. I. Ben-Zvi et al., Proc. COOL'03 Workshop (2003).

2. C. Nieter \& J.R. Cary, J. Comp. Phys. 196 (2004), p. 448.

3. Ya.S. Derbenev \& A.N. Skrinskii, Sov. J. Plasma Phys. 4 (1978), p. 273.

4. V.V. Parkhomchuk, Nucl. Instr. Meth. in Phys. Res. A 441 (2000), p. 9.

5. J. Makino \& S.J. Aarseth, Publ. Astron. Soc. Japan 44 (1992), p. 141.

6. See URL http://www.bnl.gov/RHIC/

7. B.B. Back et al. (PHOBOS collaboration), Phys. Rev. Lett. 85 (2000), p. 3100.

8. See URL http://www.agsrhichome.bnl.gov/eCool/

9. A. Fedotov et al., these proceedings.

10. Ya.S. Derbenev \& A.N. Skrinsky, Part. Accel. 8 (1978), p. 235.

11. I. Meshkov, Phys. Part. Nucl. 25 (1994), p.631.

12. Toepffer, Phys. Rev. A 66, 022714 (2002).

13. R. Busby et al., Bull. Am. Phys. Soc. 48, no. 7 (2003), p. 117.

14. G. Blelloch \& G. Narlikar, Series in Discrete Math. and Theoretical Comp. Science 30 (1997).

15. J.E. Barnes \& P. Hut, Nature 4 (1986), p. 446.

16. L. Greengard \& V. Rokhlin, J. Comp. Phys. 73 (1987), p. 325.

17. L. Greengard, The rapid evaluation of potential fields in particle systems (MIT Press, 1987).

18. J.A. Board et al., Technical Report 94-002 (Duke University, 1994).

19. J. Makino, Astrophys. Journal 369 (1991), p. 200.

20. S. Chandrasekhar, Principles of Stellar Dynamics (U. Chicago Press, 1942).

21. B.A. Trubnikov, Rev. Plasma Physics 1 (1965), p. 105.

22. NRL Plasma Formulary, ed. J.D. Huba (2000).

23. G.I. Budker, At. Energ. 22 (1967), p. 346.

24. A. Burov, FERMILAB-TM-2213 (2003). 


\title{
NUMERICAL STUDIES OF THE FRICTION FORCE FOR THE RHIC ELECTRON COOLER*
}

\author{
A.V. Fedotov ${ }^{\#}$, I. Ben-Zvi, V.N. Litvinenko, BNL, Upton, NY 11973 \\ D.L. Bruhwiler, D. Abell, R. Busby, P. Schoessow, Tech-X, Boulder, CO 80303
}

\begin{abstract}
Accurate calculation of electron cooling times requires an accurate description of the dynamical friction force. The proposed RHIC cooler will require $\sim 55 \mathrm{MeV}$ electrons, which must be obtained from an RF linac, leading to very high transverse electron temperatures. A strong solenoid will be used to magnetize the electrons and suppress the transverse temperature, but the achievable magnetized cooling logarithm will not be large. In this paper, we explore the magnetized friction force for parameters of the RHIC cooler, using the VORPAL code [1]. VORPAL can simulate dynamical friction and diffusion coefficients directly from first principles [2]. Various aspects of the friction force are addressed for the problem of highenergy electron cooling in the RHIC regime.
\end{abstract}

\section{SIMULATIONS}

The first step towards accurate calculation of cooling times is to use an accurate description of the cooling force. The achievable Coulomb logarithm in the analytic expression for the magnetized cooling force is not very large [3]. In addition, in some regimes there is a significant discrepancy between available formulas. For this reason the ParSEC project at Tech-X Corp. is being used to develop a parallel code capability based on the VORPAL code [1] to simulate from first principles the friction force and diffusion coefficients for the RHIC parameters. This project is using molecular dynamics techniques (i.e. simulating every particle in the problem) to explicitly resolve close binary collisions and thus capture the friction force and the diffusion tensor with a bare minimum of physical assumptions. Careful testing indicates that the algorithm is working well [2] for magnetized and unmagnetized cases that are relevant to the proposed electron cooling section for RHIC [4].

The primary goals of VORPAL simulation for the RHIC cooling project can be summarized as follows: 1) resolve differences in analytic calculations (approximations of uniform electron density, no space charge, infinite magnetic field, etc.); 2) determine validity of $Z^{2}$ scaling for friction force (non-linear plasma effects in magnetized plasma Debye shielding); 3) understand the effects of the space charge and diffusion dynamics; 4) understand the effects of magnetization (from strong to weak magnetization, effect of magnetic field errors); 5) accurate calculations of the friction force in the regime of small Coulomb logarithm due to magnetized collisions; 6 ) if the friction force for RHIC regime significantly deviates from description based on simple formulas, provide a numeri-

*Work supported by the U.S. Department of Energy

"fedotov@bnl.gov cal table of friction coefficients for use in other codes.

Preliminary studies with VORPAL found good agreement with available formulas in some parameters regimes and deviate in others. Some of these studies, which are directly relevant for the parameters of the magnetized cooling in RHIC are presented in this paper.

\section{MAGNETIZED FRICTION FORCE THEORY}

The process of magnetized cooling is based on the energy loss of an ion due to Coulomb interaction with the superimposed electron beam, which is guided by the longitudinal magnetic field. For a strong magnetic field, the electron dynamics in the transverse direction is effectively frozen, which together with the flattened velocity distribution of the electrons results in very fast cooling. There exist two standard approaches for the theoretical description of magnetized cooling. The first one is based on the dielectric response of the plasma to the perturbation caused by an ion. The treatment is valid for weakly coupled plasmas, and needs a carefully chosen cutoff parameter for close encounters, because linear theory cannot treat close encounters between the ion and plasma electrons. The alternative approach is based on the calculation of energy transfer due to successive binary collisions, where an approximation is needed for the shielding of the Coulomb potential by the plasma. Apart from the fact that the binary collisions approach cannot describe collective plasma excitation correctly (which may be important for some parameters), both treatments may be regarded as complementary. In fact, similar results can be achieved with the appropriate choice of the cutoff parameter $[5,6]$.

Closed form analytic expressions can be obtained only in the limiting cases of zero and infinite magnetic field, with numerical simulations required for finite magnetic field values. A simplified kinetic model can be described by considering two types of collisions: 1) fast collisions, where effective interaction time is small compared to the Larmor period of electrons; 2) adiabatic (magnetized) collisions, where interaction time is much longer than the Larmor period. As a result, the friction force can be approximated as a non-magnetized part (fast collision) and magnetized part (adiabatic collision) as described by Derbenev and Skrinsky (D-S) [7]. The relative role of fast and adiabatic collisions depends on the relative velocity of ions. The general treatment results in three regions of the impact parameters and relative ion velocities (with an additional repetitive type of collisions also being included). In such a form, suitable for cooling dynamics simulations, the friction force formulas were presented by Meshkov [8], and are sometimes referred to as Derbenev- 
Skrinksy-Meshkov (D-S-M) formulas. In contrast, Parkhomchuk presents a parametric formula - a generalization of the unmagnetized result, which is predicted [9] to agree reasonably well with experiments for a wide range of parameters. The Derbenev-Skrinsky-Meshkov (D-S, D-S-M) [7, 8] and Parkhomchuk (VP) [9] formulas disagree strongly in some parameter regimes, while showing approximate (roughly factor of two) agreement in other regimes. For completeness and comparison with simulations these formulas are listed below. The magnetized part of the Derbenev-Skrinky (D-S) formulas [7] is given by the following expressions:

$$
\begin{aligned}
& \mathrm{F}_{\perp}^{D S}=-\frac{1}{2} \omega_{p e}^{2} \frac{(Z e)^{2}}{4 \pi \varepsilon_{0}} \Lambda^{A}\left(V_{i o n}\right) \frac{\left(V_{\perp}^{2}-2 V_{\|}^{2}\right)}{V_{i o n}^{2}} \frac{V_{\perp}}{V_{i o n}^{3}} \\
& \mathrm{~F}_{\|}^{D S}=-\frac{3}{2} \omega_{p e}^{2} \frac{(Z e)^{2}}{4 \pi \varepsilon_{0}}\left[\Lambda^{A}\left(V_{i o n}\right)\left(\frac{V_{\perp}}{V_{i o n}}\right)^{2}+\frac{2}{3}\right] \frac{V_{\|}}{V_{i o n}^{3}}
\end{aligned}
$$

for $V_{\text {ion }} \gg \Delta_{\text {eII }}$ (longitudinal rms velocity of electrons) and:

$$
\begin{aligned}
& \mathrm{F}_{\perp}^{D S}=-\frac{1}{\sqrt{2 \pi}} \omega_{p e}^{2} \frac{(Z e)^{2}}{4 \pi \varepsilon_{0}} \Lambda^{A}\left(V_{\perp}\right) \frac{V_{\perp}}{\Delta_{e\|\|}^{3}} \\
& \mathrm{~F}_{\|}^{D S}=-\frac{1}{\sqrt{2 \pi}} \omega_{p e}^{2} \frac{(Z e)^{2}}{4 \pi \varepsilon_{0}} \Lambda^{A}\left(\Delta_{e \|}\right) \frac{V_{\|}}{\Delta_{e \|}^{3}}
\end{aligned}
$$

for $V_{\text {ion }}<\Delta_{\text {ell }}$, where

$$
\begin{aligned}
& \Lambda^{A}=\ln \left(\rho_{\max } / \rho_{\min }\right) \quad V_{i o n}^{2}=V_{\|}^{2}+V_{\perp}^{2} \\
& \rho_{\min }^{D S}=\max \left(r_{L}, \rho_{\min }\right) \rho_{\max }^{D S}=V_{r e l}^{D S} / \max \left(\omega_{p e}, 1 / \tau\right) \\
& r_{L}=V_{r m s, e, \perp} / \Omega_{L} \quad V_{r e l}^{D S}=\max \left(V_{i o l}, \Delta_{e \|}\right)
\end{aligned}
$$

The Parkhomchuk's (VP) empiric formula is [9]:

$$
\begin{gathered}
\mathrm{F}^{V P}=-\frac{1}{\pi} \omega_{p e}^{2} \frac{(Z e)^{2}}{4 \pi \varepsilon_{0}} \Lambda^{M} \frac{\mathrm{V}_{\text {ion }}}{\left(V_{\text {ion }}^{2}+V_{e f f}^{2}\right)^{3 / 2}} \\
\Lambda^{M}=\ln \left(\frac{\rho_{\max }^{P}+\rho_{\min }^{P}+r_{L}}{\rho_{\min }^{P}+r_{L}}\right) \rho_{\max }^{P}=V_{i o n} / \max \left(\omega_{p e}, 1 / \tau\right) \\
\rho_{\min }^{P}=\left(2 \mathrm{~b} e^{2} / 4 \pi \varepsilon_{0}\right) / m_{e} V_{i o n}^{2} \quad V_{e f f}^{2}=\Lambda_{e \| l}^{2}+\Delta V_{\perp e}^{2}
\end{gathered}
$$

Parkhomchuk's formula includes an effective velocity which may help to relate formulas to realistic situations for experiments. Magnetized cooling is governed by the longitudinal rms velocity spread of electrons $\Delta_{\mathrm{eII}}$ (since transverse motion is effectively suppressed), which can be a very small quantity, ensuring "fast cooling". However, in real life, the spread of electrons can be increased due to various effects such as imperfection of the solenoidal field or the space-charge of the electron beam in the cooling section, for example. Their effect can be parametrically described with an additional contribution to an rms veloc- ity spread $\left(\Delta \mathrm{V}_{\mathrm{e}}\right)$, which results in the definition of $\mathrm{V}_{\text {eff }}$ in Eq. (2c) [9].

Order-of-magnitude agreement between various magnetized friction force formulas has been acceptable for existing electron cooling facilities, because they operate in a regime where the friction force is strong enough by a large margin. In contrast, the proposed RHIC cooler will operate in a completely new regime, where the estimated friction force is expected to be adequate but with little margin for error [10]. As a result, we are trying to understand the accuracy of available formulas for various degrees of magnetization, based on numerical simulations with the VORPAL code.

\section{NUMERICAL STUDIES}

Although detailed benchmarking of the VORPAL code vs theory and experimental data is still in progress, recent advances in code development [2] allowed us to begin systematic study of the magnetized friction force for parameters of the RHIC cooler. As a result of aggressive beam dynamics studies (both of magnetized beam transport and cooling dynamics), the design parameters are also evolving.

Various design solutions are being studied to address technical issues and possible effects of cooling on ion beam dynamics. For example, it is technically difficult to produce one long superconducting solenoid of length $\mathrm{L}=30$ or 40 meters. To compensate for coupling (introduced by a solenoid) it may be beneficial to have half-length solenoids with reversed magnetic fields. However, this would result in a reduction of magnetized Coulomb logarithm. As a result, the optimum length of solenoid section (as well as total number of such sections) is presently under study [3]. Similar discussion is true for field strength in the solenoid with present consideration in the range of $\mathrm{B}=2-5 \mathrm{~T}$ (depending on the achievable emittance for magnetized transport of electron bunches with very high charge), density of electrons in the range $\mathrm{n}_{\mathrm{e}}=0.5-2 \times 10^{15} \mathrm{~m}^{-3}$ (depending on several effects).

As a result, validity of available formulas should be established with VORPAL for a wide range of parameters, to resolve many outstanding issues. Detailed study for RHIC parameters will be reported later elsewhere. Here, we choose the upper end of RHIC parameters to show capabilities of the code. The parameters chosen for simulations are the following (in the beam moving frame): $\mathrm{Au}$ ions with $\mathrm{Z}=79$, time of flight through the solenoid $\tau=0.4 \mathrm{~ns}$ (length of solenoid section $L=13 \mathrm{~m}$ ), magnetic field $\mathrm{B}=5 \mathrm{~T} ; n_{\mathrm{e}}=2 \times 10^{15} \mathrm{~m}^{-3}$, transverse rms spread of electrons $\Delta_{\mathrm{e}, \perp}=8 \times 10^{6} \mathrm{~m} / \mathrm{s}$ (which corresponds to typical parameters of the $20 \mathrm{nC}$ magnetized electron beam with transverse normalized rms emittance of $30 \mu \mathrm{m})$, longitudinal rms spread of electrons $\Delta_{\mathrm{e}, \|}=1 \times 10^{5} \mathrm{~m} / \mathrm{s}$ (corresponds to an $\mathrm{rms}$ momentum spread of $\left.3^{*} 10^{-4}\right)$, and $V_{\text {eff }}=\Delta_{\text {eII }}$ (no solenoid imperfections).

Figure 1 shows dependence of the longitudinal component of the friction force on ion velocity for ion motion along the magnetic field lines (zero component of the 
transverse ion velocity): 1) Eq. (1b,d) (blue curve in two regions of applicability) 2) Eq. (2) (green dotted curve) 3) VORPAL results (pink dots with error bars). For these parameters, one can see tendency of formula in Eq. (2) to underestimate numerical factor for the friction force compared to numerical results.

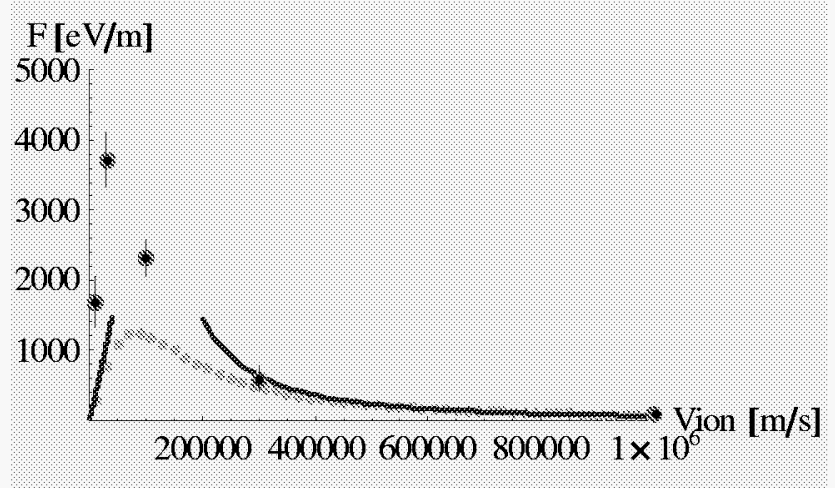

Figure 1: Dependence of the longitudinal component of the friction force on ion velocity: 1) Eq. (1b,d) (blue curve in two regions) 2) Eq. (2) (green dotted curve) 3) VORPAL results (pink dots with error bars).

For realistic simulations of magnetized cooling dynamics [10], it is very important to accurately capture the friction force for ion trajectories at different angles with respect to the magnetic field lines. Equations (1) and (2) show dramatic differences in this respect. Equations (1) show anisotropy introduced by a strong magnetic field, while Eq.'s (2) neglect such behavior. In fact, the main argument in Ref. [9] in favor of Eq. (2a) was that Eq. (1b) gives zero results for the longitudinal component of the friction force when the transverse component of ion velocity goes to zero. This happens only if the logarithmic term is kept in Eq. (1b), while the constant term is neglected. However, the magnetized Coulomb logarithm typically has a small value of around 2-3 (even smaller for small relative velocities), so that for small transverse angles $\left(\sin (\theta)=\mathrm{V}_{\text {ion,tr }} / \mathrm{V}_{\text {ion }}\right)$, the constant and logarithmic terms in Eq. (1b) become comparable.

Figure 2 shows the dependence of the longitudinal component of the friction force on the transverse angle $\theta$ for simulation parameters close to those described above, and fixed ion velocity of $3^{*} 10^{5} \mathrm{~m} / \mathrm{s}$. The conditions of applicability for Eq. (1b) are satisfied: 1) red curve - Eq (1b) with a constant term being neglected (as used in [9]); 2) blue curve - Eq. (1b) with both the logarithmic and constant terms; 3) green curve - Eq. (2); 4) pinks dots with error bars - VORPAL results. One may argue about accuracy and validity of the formulas derived in logarithmic approximation, but we see a remarkable agreement with the direct numerical simulation obtained with VORPAL, which are not based on these logarithms or any analytic approximations.

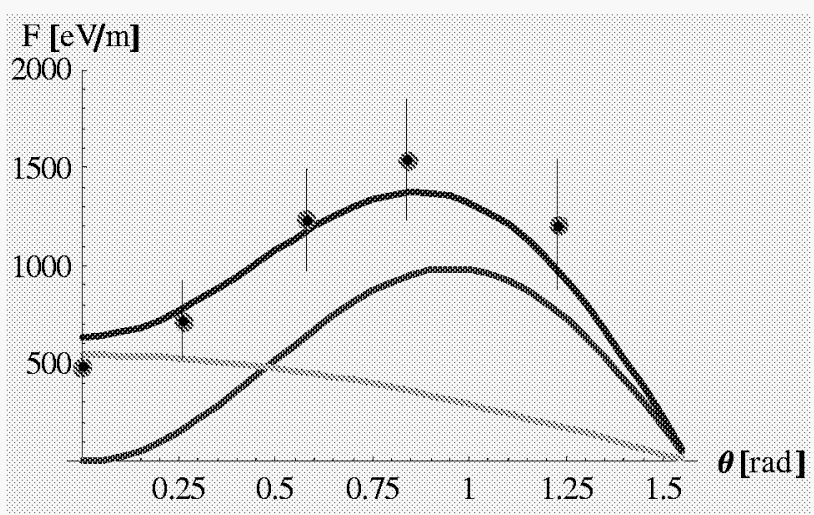

Figure 2: Dependence of the longitudinal component of the friction force on the transverse angle of ion velocities - comparison of formulas vs direct numeric simulations.

The on-going simulations with the VORPAL code show great promise for resolving ambiguities in the theoretical understanding of the magnetized friction force under idealized conditions, and also for determining quantitatively the effect of complicating factors, including the impact of errors in the magnetic field [11]. The benchmarking of the code vs recent precision measurements of the friction force at CELSIUS cooler [12] is also in progress and will be reported later.

\section{ACKNOWLEDGMENTS}

We would like to thank A. Burov, Ya. Derbenev, J. Kewisch, A. Sidorin, and the Accelerator Physics Group of Electron Cooling Project of RHIC for many useful discussions during these studies.

\section{REFERENCES}

[1] C. Nieter, J. Cary, J. Comp. Phys. 196, p. 448 (2004).

[2] D. Bruhwiler et al., Proceedings of ICFA-HB2004 Workshop, Bensheim, Germany (2004).

[3] RHIC E-cooler Design Report

$\mathrm{http} / /$ www.agsrhichome.bnl.gov/eCool

[4] A. V. Fedotov et al., TPAT089, these proceedings.

[5] N.S. Dikansky et al., BINP preprint 88-61 (1988).

[6] H.B. Nersisyan, G. Zwicknagel, C. Toepffer, Phys. Rev. E 67, 026411 (2003), and references therein

[7]Ya. Derbenev, A. Skrinsky, Part. Acc. 8, p. 235 (1978);

Ya. Derbenev, A. Skrinsky, Sov. Ph. Rev 1, 165 (1981).

[8] I. Meshkov, Phys. Part. Nucl. 25, p.631 (1994), and references therein.

[9] V. Parkhomchuk, Nucl. Inst. Meth. A 441, p. 9 (2000).

[10] A.V. Fedotov et al., TPAT090, these proceedings.

[11] D. Bruhwiler et al., TPAT087, these proceedings.

[12] A. Fedotov, B. Galnander, V. Litvinenko, T. Lofnes, A. Sidorin, A. Smirnov, V. Ziemann, "Experiments towards high-energy cooling", in preparation. 


\title{
THE EFFECT OF MAGNETIC FIELD ERRORS ON DYNAMICAL FRICTION IN ELECTRON COOLERS*
}

\author{
D.L. Bruhwiler," R. Busby, D.T. Abell, S. Veitzer, Tech-X Corp., Boulder, CO 80303, U.S.A. \\ A.V. Fedotov, V.N. Litvinenko, BNL, Upton, NY 11973, U.S.A.
}

\begin{abstract}
A proposed luminosity upgrade to the Relativistic Heavy Ion Collider (RHIC) includes a novel electron cooling section [1], which would use $\sim 55 \mathrm{MeV}$ electrons to cool fully-ionized $100 \mathrm{GeV} /$ nucleon gold ions. A strong (1-5 T) solenoidal field will be used to magnetize the electrons and thus enhance the dynamical friction force on the ions. The physics of magnetized friction is being simulated for RHIC parameters [2,3,4], using the VORPAL code [5]. Most theoretical treatments for magnetized dynamical friction do not consider the effect of magnetic field errors, except in a parametric fashion. However, field errors can in some cases dramatically reduce the velocity drag and corresponding cooling rate. We present two simple analytical models for the magnetic field errors. The simulated dynamical friction for the case of a perfect solenoidal field is compared with results from these new models. We simulate parameters for the existing cooler of the CELSIUS ring, because recent experiments [6] provide data that will later be used for code validation.
\end{abstract}

\section{MOTIVATION}

The semi-analytic simulation codes SIMCOOL [7] and BETACOOL [8] are being used [3,9] to design the proposed RHIC cooler. These codes rely on analytical expressions for the friction force on a single ion moving through a magnetized electron distribution. In particular, the formulas from Meshkov (DSM) [10] and Parkhomchuk (VP) [11] are generally used. The Meshkov result is a modification of previous work by Derbenev and Skrinsky (DS) [12]. The VP formula is an empirical generalization of the theoretical friction force for unmagnetized electrons. The semi-numerical results of Toepffer [13] are also interesting.

These calculations and parameterizations assume a constant electron density, a uniform neutralizing background, the absence of any perturbation from other ion trajectories, and a constant longitudinal magnetic field. The VP formula includes an effective velocity which may help to remove some of these assumptions in a parametric fashion. In this paper, we present work in progress, with the goal of understanding the effects of magnetic field errors. The DS and VP equations are reproduced elsewhere in this proceedings [4].

\section{MAGNETIC FIELD ERRORS}

We consider two simple models for errors in the field of a solenoid magnet, neglecting fringe field regions. Both

\footnotetext{
* Work supported by U.S. DOE under grant DE-FG03-01ER83313. \# Trabiwile@troorp.com
}

models are current-free and divergence-free, for the most part. One is a sum of terms that vary sinusoidally along the longitudinal axis and exponentially in the transverse plane. The other is a piece-wise constant series of small tilts in the magnetic field direction. We assume for both models that, to lowest order, we have a constant magnetic field in the $\mathrm{z}$ direction.

The first model uses separation of variables to obtain:

$$
\begin{aligned}
& B_{x}=\sum_{i} b_{i} \frac{k_{x, i}}{k_{z, i}} \exp \left(k_{x, i} x\right) \exp \left(k_{y, i} y\right) \sin \left(k_{z, i} z+\varphi_{z, i}\right) \\
& B_{y}=\sum_{i} b_{i} \frac{k_{y, i}}{k_{z, i}} \exp \left(k_{x, i} x\right) \exp \left(k_{y, i} y\right) \sin \left(k_{z, i} z+\varphi_{z, i}\right) \\
& B_{z}=B_{0}+\sum_{i} b_{i} \exp \left(k_{x, i} x\right) \exp \left(k_{y, i} y\right) \cos \left(k_{z, i} z+\varphi_{z, i}\right) \\
& k_{z, i}^{2}=k_{x, i}^{2}+k_{y, i}^{2} \quad \lambda_{i}=2 \pi / k_{z, i}
\end{aligned}
$$

where $B_{0}$ is the idealized solenoid field, and we assume $b_{i}<<B_{0}$ for all $i$. In general, the terms like $\exp \left(k_{x, i} x\right)$ can be replaced by a sum of two exponentials, one with postive and one with negative argument, or equivalently by a sum of $\sinh$ and $\cosh$ terms. It's also possible to replace one (but not both) of the exponential terms (for every i) as a sum of sinusoidal terms. However, in this latter case, one must modify Eq. (1d) with an appropriate sign change.

The second model is motivated by the characteristics of the solenoid field in the electron cooler of the CELSIUS ring in Sweden. Figure 3 of Ref. [14] shows that, ignoring fringe fields, deviations of the magnetic field from that of an ideal solenoid can be approximated by a sum of piecewise constant tilts, of the general form:

$$
\begin{aligned}
& B_{x}=\sum_{i} b_{x, i} H\left(z-z_{x, i}\right) H\left(z_{x, i+1}-z\right) \\
& B_{y}=\sum_{i} b_{y, i} H\left(z-z_{y, i}\right) H\left(z_{y, i+1}-z\right) \\
& B_{z}=B_{0}
\end{aligned}
$$

where $H(x)$ is the unit Heaviside function, and we assume $b_{x, i}, b_{y, i}<<B_{0}$ for all $i$. This model has the disadvantage of (relatively small) violations of Maxwell's equations wherever $z=z_{x, i}$ or $z_{y ; i}$. However, it would require many terms for Eq.'s (1) to reasonably model the characteristics of the magnetic field errors seen in [14], while Eq's (2) can do so simply and efficiently.

In order to apply these field errors in our VORPAL simulations, we must Lorentz transform them to the beam frame, which we take to be moving along the $z$-axis with 
velocity $\beta c$ and relativistic factor $\gamma$. Using the standard Lorentz transforms of fields and coordinates [15] for the case where $E=O$ in the lab frame, we obtain the following:

$$
\begin{aligned}
& B_{z}{ }^{\prime}=B_{z}\left(x^{\prime}, y^{\prime}, \gamma \beta c t^{\prime}\right) \\
& B_{x}{ }^{\prime}=\gamma B_{x}\left(x^{\prime}, y^{\prime}, \gamma \beta c t^{\prime}\right) \quad B_{y}{ }^{\prime}=\gamma B_{y}\left(x^{\prime}, y^{\prime}, \gamma \beta c t^{\prime}\right) \\
& E_{x}{ }^{\prime}=-\beta c B_{y}{ }^{\prime} \quad E_{y}{ }^{\prime}=\beta c B_{x}{ }^{\prime} \quad E_{z}{ }^{\prime}=0
\end{aligned}
$$

where the primes indicate beam frame quantities. Strictly speaking, Eq.'s (3) should also include a $z$ ' dependence, but we can ignore this, because the simulations consider only a small domain with periodic boundary conditions.

\section{SIMULATION DETAILS}

We have developed, within the VORPAL code, a 3D algorithm capable of directly simulating from first principles the magnetized (or unmagnetized) friction force and diffusion coefficients for ions in an electron (or positron) cooler. This algorithm uses molecular dynamics techniques (i.e. simulating every particle in the problem) to explicitly resolve close binary collisions and thus capture the friction force and the diffusion tensor with a bare minimum of physical assumptions [2,16]. This required generalization of the $4^{\text {th }}$-order algorithm of Makino \& Aarseth [17] to accommodate charged particles in a magnetic field.

\section{Parameters Used for the CELSIUS Cooler}

Relevant physical and numerical parameters are shown in Table 1. Most physical quantities are not known precisely. The longitudinal rms velocity of the electrons in the cooler is $\Delta_{e, I I} \sim 3000 \mathrm{~m} / \mathrm{s}$. In the absence of any model for magnetic field errors, we attempt to capture the associated effects in a parametric fashion by increasing this rms velocity to an effective value of $\Delta_{\text {eff, }}=9000 \mathrm{~m} / \mathrm{s}$.

These periodic electrostatic simulations are conducted in the beam frame, where all particle motion is non-relativistic, within a domain (typically a cube) just large enough to capture the largest relevant impact parameters. The electrons interact only with the ions (typically eight of them), and not with each other, which reduces the runtime by factors of order $10^{5}$, at the expense of losing correct e- shielding dynamics. For RHIC parameters, the interaction time in the cooler is shorter than the plasma period, so this approximation is justified. For CELSIUS parameters, this approximation should be validated.

\section{Cluster Computing with VORPAL}

The ion dynamics during a single pass through the cooler is strongly dominated by diffusion [2], making it very difficult to extract the average velocity drag due to dynamical friction with any precision. Diffusion cannot be suppressed through the numerical trick of using correlated e-/e+ pairs [2], because for CELSIUS parameters the friction due to positrons is very different. Hence, the only recourse is to repeat a given simulation many times, each time with different seeds for generating the initial electron

positions and velocities. This allows one to quote the average friction force (or velocity drag) with meaningful statistical error bars.

Managing 100's of VORPAL simulations for each parameter set is made tractable through use of the TaskDLiSH software package, which provides task farm functionality for executing shell scripts on a cluster. TaskDLiSH, a simplified implementation of TaskDL [18], works by first creating a "tuple space," in which tasks are placed. The tasks here are simply shell scripts, which launch VORPAL with slightly modified input parameters.

Table 1: Simulation Parameters for CELSIUS Cooler

\begin{tabular}{|c|c|l|c|}
\hline Symbol & \multicolumn{1}{|c|}{ Meaning } & \multicolumn{1}{|c|}{ Value } & Units \\
\hline $\mathrm{B}_{0}$ & solenoid field & 0.1 & $\mathrm{~T}$ \\
\hline $\mathrm{L}_{\mathrm{sol}}$ & solenoid length & 2.5 & $\mathrm{~m}$ \\
\hline$\beta$ & proton bunch velocity / c & 0.308 & \\
\hline$\tau_{\text {lab }}$ & interaction time (lab frame) & $2.7 \times 10^{-8}$ & $\mathrm{~s}$ \\
\hline$\tau_{\text {beam }}$ & interact. time (beam frame) & $2.6 \times 10^{-8}$ & $\mathrm{~s}$ \\
\hline$\Delta \mathrm{t}$ & largest time step & $2.6 \times 10^{-12}$ & $\mathrm{~s}$ \\
\hline $\mathrm{dt}_{\mathrm{min}}$ & smallest time step & $8.0 \times 10^{-14}$ & $\mathrm{~s}$ \\
\hline$\omega_{\mathrm{pe}}$ & e- plasma frequency & $4.1 \times 10^{8}$ & $\mathrm{rad} / \mathrm{s}$ \\
\hline$\Omega_{\mathrm{L}}$ & e- Larmor frequency & $1.8 \times 10^{10}$ & $\mathrm{rad} / \mathrm{s}$ \\
\hline $\mathrm{r}_{\mathrm{L}}$ & e- Larmor (gyro-) radius & $7.9 \times 10^{-6}$ & $\mathrm{~m}$ \\
\hline $\mathrm{L}_{\mathrm{x}, \mathrm{y}, \mathrm{z}}$ & sim. domain dimensions & $6.0 \times 10^{-4}$ & $\mathrm{~m}$ \\
\hline $\mathrm{n}_{\mathrm{e}}$ & e- number density & $5.4 \times 10^{13}$ & $\mathrm{~m}{ }^{-3}$ \\
\hline $\mathrm{N}_{\mathrm{e}}$ & \# of simulated e-'s & $1.2 \times 10^{3}$ & \\
\hline$\Delta_{\mathrm{e}, \perp}$ & transverse rms e- velocity & $1.4 \times 10^{5}$ & $\mathrm{~m} / \mathrm{s}$ \\
\hline$\Delta_{\mathrm{e}, \mathrm{ll}}$ & long. rms e- velocity & $3.0 \times 10^{3}$ & $\mathrm{~m} / \mathrm{s}$ \\
\hline$\Delta_{\mathrm{eff}, \|}$ & effective long. rms e- vel. & $9.0 \times 10^{3}$ & $\mathrm{~m} / \mathrm{s}$ \\
\hline
\end{tabular}

Once the tuple space is created, distributed "workers" launch the tasks on remote. Workers then reserve and process tasks independently. New tasks may be added to the tuple space at any time, based on the results of previously executed tasks, and workers may be managed (e.g. decommissioned) by placing directed tasks into the workspace. TaskDLiSH provides efficient scheduling of cluster resources and automated load balancing, because workers poll for tasks when they are ready. Fault tolerance is automatic, because the failure of one worker or task does not impact other workers or tasks.

\section{STATUS \& FUTURE PLANS}

We have conducted many simulations for CELSIUS parameters, using the higher effective rms electron 
velocity $\Delta_{\text {eff, } \|}=9000 \mathrm{~m} / \mathrm{s}$, with no magnetic field errors. From one to five thousand ion trajectories were generated in each case, in order to extract from the diffusion dominated dynamics a reasonable average value for the velocity drag. This work will be presented elsewhere.

We have begun to repeat some of these simulations with the expected rms electron velocity $\Delta_{e, \|}=3000 \mathrm{~m} / \mathrm{s}$, including magnetic field errors as described by the "first model" of Eq.'s (1) or the "second model" of Eq.'s (2). For the second model, we are using a series of terms taken directly from the mapped field errors of the CELSIUS solenoid. For the first model, we are at present considering only a single sinusoidal term, symmetric in $x$ and $y$, with an amplitude $\left(\mathrm{b}_{1}=0.001 * \mathrm{~B}_{0}\right)$ and wavelength $(20 \mathrm{~cm})$ that are roughly consistent with the CELSIUS solenoid measurements.

Initial results indicate that the second model will suppress the velocity drag (i.e. reduce the friction force and thus increase the cooling time) more strongly than the first model with a single term. We don't present details here, because the simulation results must be examined further and many more ion trajectories must yet be generated. Also, it remains an open question how to properly choose the number of sinusoidal terms, much less their amplitudes, wavelengths and degrees of asymmetry.

In future work, simulation results will be validated through comparison with recently obtained data [6]. Also, the understanding and expertise gained during study of CELSIUS parameters, will later be applied to the parameters of the proposed RHIC cooler.

\section{ACKNOWLEDGEMENTS}

The authors thank I. Ben-Zvi, J.R. Cary, P. Messmer, P.V. Schoessow, P. Stoltz, V. Yakimenko, and the Accelerator Physics Group of the RHIC Electron Cooling Project for many helpful discussions. Discussions with A.K. Jain regarding solenoid field errors were especially helpful. This work was supported by the U.S. Dept. of Energy under grant DE-FG03-01ER83313 and by Tech-X Corp. We used resources of NERSC, which is supported by the U.S. Dept. of Energy under contract DE-AC03$76 \mathrm{SF} 00098$.

\section{REFERENCES}

[1] I. Ben-Zvi et al., TPAP043, these proceedings.

[2] D.L. Bruhwiler et al., Proc. 33 ${ }^{\text {rd }}$ ICFA Advanced Beam Dynamics Workshop (2004), in press.

[3] A.V. Fedotov et al., Proc. $33^{\text {rd }}$ ICFA Advanced Beam Dynamics Workshop (2004), in press.

[4] A.V. Fedotov et al., TPAT092, these proceedings.

[5] C. Nieter and J.R. Cary, J. Comp. Phys. 196, 448 (2004).

[6] A. Fedotov, B. Galnander, V. Litvinenko, T. Lofnes, A. Sidorin, A. Smirnov, V. Ziemann, "Experiments towards high-energy cooling", in preparation.

[7] Yu. Eidelman and A.V. Fedotov, "Recent developments of the SIMCOOL code," unpublished.

[8] See URL http://lepta.in xubetacoul fHes'trame hom

[9] A.V. Fedotov et al., TPAT090, these proceedings.

[10]I. Meshkov, Phys. Part. Nucl. 25, 631 (1994).

[11] V. Parkhomchuk, Nucl. Instr. Meth. Phys. Res. A 441, 9 (2000).

[12] Ya.S. Derbenev and A.N. Skrinsky, Part. Accel. 8, 235 (1978); Sov. Phy. Rev. 1, 165 (1981).

[13]C. Toepffer, Phys. Rev. A 66, 022714 (2002).

[14]M. Sedlacek et al., "Design and Construction of the CELSIUS Electron Cooler," Workshop on Beam Cooling (CERN, 1994); httpifpreprintscernchicgibinisellink?base=cemrep\&eateg=Yellow Report\&id $=9403$

[15] J. Jackson, Classical Electrodynamics, $2^{\text {nd }}$ Ed. (John Wiley \& Sons, New York, 1975).

[16]R. Busby et al., Bull. Am. Phys. Soc. 48, no. 7 (2003), p. 117.

[17] J. Makino \& S.J. Aarseth, Publ. Astron. Soc. Japan 44 (1992), p. 141

[18] A description of TaskDL can be found on-line at

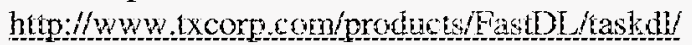




\section{Tech-X Corporation}

Final Report: DE-FG03-01ER83313

\section{Estimate of Technical and Economic Feasibility}

Tech-X will pursue contract opportunities with accelerator laboratories around the world in electron cooling physics and related research areas..

\section{References}

1 The official RHIC web site at URL http://www.rhic.bnl.gov/

2 I. Ben-Zvi et al., "Luminosity Upgrade Options for RHIC," (May, 2001), RHIC Note \# CA/AP/51; see URL http://www.agsrhichome.bnl.gov/AP/ap_notes/ap_note_51.pdf

3 The Fermilab electron cooling web page at URL http://www-ap.fnal.gov/ecool/

4 The BETACOOL web site at URL http://lepta.jinr.ru/index.htm

5 A. Sidorin, "BETACOOL: New features \& developments," presented at BNL (Dec., 2003); see

URL http://www.agsrhichome.bnl.gov/eCool/workshop1203/3_Sidorin_BETACOOL.pdf

6 V. Parkhomchuk and I. Ben-Zvi, "Electron Cooling for RHIC - Preliminary Review of the Principles of Electron Cooling for the Relativistic Heavy Ion Collider," (April, 2001), RHIC Note \# $\quad$ C-A/AP/47; see URL http://www.agsrhichome.bnl.gov/AP/ap_notes/ap_note_47.pdf

7 Yu. Eidelman, "SimCool: Recent Developments," presented at BNL (Dec. 2003); see URL http://www.agsrhichome.bnl.gov/eCool/workshop1203/4_Eidelman_simcool.pdf

8 A.V. Fedotov, I. Ben-Zvi, D.L. Bruhwiler, Yu. Eidelman, V.N. Litvinenko, N. Malitsky, I. Meshkov, A. Sidorin, A. Smirnov, G. Trubnikov, "Electron Cooling Dynamics for RHIC," in High Intensity and High Brightness Hadron Beams, Ed. by I. Hofmann, R.W. Hasse and J.-M. Lagniel, AIP Conf. Proc. 773 (2005), p. 415.

9 C. Nieter \& J.R. Cary, J. Comp. Phys. 196 (2004), p. 448.

10 Ya.S. Derbenev \& A.N. Skrinskii, Sov. J. Plasma Phys. 4 (1978), p. 273.

11 V.V. Parkhomchuk, Nucl. Instr. Meth. in Phys. Res. A 441 (2000), p. 9.

12 D.L. Bruhwiler, R. Busby, D.T. Abell, S. Veitzer, A.V. Fedotov and V.N. Litvinenko., "The Effect of Magnetic Field Errors on Dynamical Friction in Electron Coolers," in Proc. Particle Accel. Conf. (2005), in press.

13 A.V. Fedotov, I. Ben-Zvi, V.N. Litvinenko, D.L. Bruhwiler, D.T. Abell, R. Busby and P. Schoessow, "Numerical Studies of the Friction Force for the RHIC Electron Cooler," in Proc. Particle Accel. Conf. (2005), in press.

14 D.L. Bruhwiler, R. Busby, A.V. Fedotov, I. Ben-Zvi, J.R. Cary, P. Stoltz, A. Burov, V.N. Litvinenko, P. Messmer, D.T. Abell, C. Nieter, "Direct simulation of friction forces for heavy ions interacting with a warm magnetized electron distribution," in High Intensity and High Brightness Hadron Beams, Ed. by I. Hofmann, R.W. Hasse and J.-M. Lagniel, AIP Conf. Proc. 773 (2005), p. 394. 
15 R. Busby, D.L. Bruhwiler, P. Stoltz, D.T. Abell, J.R. Cary, P. Messmer, I. Ben-Zvi and A. Burov, "Direct Simulation of Friction and Diffusion Coefficients for Ions Interacting with a Magnetized Electron Distribution," Bull. Am. Phys. Soc. 48, no. 7 (2003), p. 117.

16 D.L. Bruhwiler, R. Busby, D.T. Abell, P. Messmer, P. Stoltz, J.R. Cary, A. Fedotov, I. BenZvi, V. Litvinenko and A. Burov, "Simulating Electron Cooling Physics with VORPAL Status, Current Plans \& Future Developments," presented at BNL (Dec., 2003); see URL http://www.agsrhichome.bnl.gov/eCool/workshop1203/2_Bruhwiler_VORPAL_status.pdf

17 D.L. Bruhwiler, R. Busby, D.T. Abell, P. Messmer, P. Stoltz, J.R. Cary, A. Fedotov, I. BenZvi, V. Litvinenko and A. Burov, "Simulating Electron Cooling Physics with VORPAL Recent Results," presented at BNL (Dec., 2003); see URL http://www.agsrhichome.bnl.gov/eCool/workshop1203/6_Bruhwiler_VORPAL_results.pdf

18 J. Makino \& S.J. Aarseth, Publ. Astron. Soc. Japan 44 (1992), p. 141.

19 B.B. Back et al. (PHOBOS collaboration), "Charged particle multiplicity near mid-rapidity in central $\mathrm{Au}+\mathrm{Au}$ collisions sqrt(s) $=56$ and $130 \mathrm{AGeV}$," Phys. Rev. Lett. 85 (2000), p. 3100 .

20 I. Ben-Zvi, "Electron Cooling for RHIC," presented at ECOOL workshop (2001); see URL http://www.agsrhichome.bnl.gov/eCool/pap_ecool01.pdf

21 The Brookhaven electron cooling web page at URL http://www.agsrhichome.bnl.gov/eCool/

22 I. Ben-Zvi et al., "Electron Cooling for RHIC," Proc. IEEE Part. Accel. Conf. (2001), p. 48.

23 S. Nagaitsev et al., "Status of the Fermilab Electron Cooling Project," Proc. 1999 Particle Accel. Conf. (New York, 1999), p. 521.

24 G. Budker, Sov. J. Atomic Energy 22 (1967), p. 346.

25 T. Ellison et al., "Cooled Beam Intensity Limits in the IUCF Cooler," in Proc. Workshop on Beam Cooling and Related Topics (Montreux, October, 1993), CERN 94-03, p. 377.

26 M. Steck et al., "Electron Cooling Assisted Beam Accumulation in the Heavy Ion Synchrotron SIS by Repeated Multiturn Injection," Proc. 1999 Particle Accel. Conf. (New York, 1999), p. 1704.

27 T. Tanabe et al., "Development of MUSES-ACR Electron Cooler," Proc. 1999 Particle Accel. Conf. (New York, 1999), p. 1722.

28 P. Messmer and D.L. Bruhwiler, "A parallel electrostatic solver for the VORPAL code," Comp. Phys. Comm. 164 (2004), p. 118.

29 D.L. Bruhwiler, S.G. Shasharina and J.R. Cary, Computing in Object Oriented Parallel Environments, Lecture Notes in Computer Science 1732 (1999), pp. 154-163.

30 VORPAL at Univ. of Colorado; http://www-beams.colorado.edu/vorpal/

31 VORPAL at Tech-X Corp.; http://www.txcorp.com/technologies/VORPAL/

32 "Dream Beam" issue; http://www.nature.com/nature/links/040930/040930-1.html

33 C.G.R. Geddes, Cs. Toth, J. van Tilborg, E. Esarey, C.B. Schroeder, D.L. Bruhwiler, C. 


\section{Tech-X Corporation}

Nieter, J.R. Cary, and W.P. Leemans, "High-quality electron beams from a laser wakefield accelerator using plasma-channel guiding," Nature 431 (2004), p. 538.

34 G. Blelloch and G. Narlikar, "A Practical Comparison of N-Body Algorithms," Parallel Algorithms, Series in Discrete Mathematics and Theoretical Computer Science 30 (1997).

35 J.E. Barnes and $P$. Hut, "A hierarchical $O(N \log N)$ force calculation algorithm," Nature 4 (1986), p. 446.

36 L. Greengard and V. Rokhlin, "A fast algorithm for particle simulation," J. of Comp. Phys. 73 (1987), p. 325.

37 L. Greengard, The rapid evaluation of potential fields in particle systems (MIT Press, 1987).

38 J.A. Board, Z.S. Hakura, W.S. Elliot, D.C. Gray, W.J. Blanke and J.F. Leathrum Jr., "Scalable implementations of multipole-accelerated algorithms for molecular dynamics," Technical Report 94-002 (Duke University, 1994). 